\title{
Color Badger: A Novel Retinex-Based Local Tone Mapping Operator
}

\author{
Nikola Banić and Sven Lončarić \\ Image Processing Group \\ Department of Electronic Systems and Information Processing \\ Faculty of Electrical Engineering and Computing \\ University of Zagreb, Croatia \\ $\{$ nikola.banic, sven.loncaric $\} @ f e r . h r$ \\ http://www.fer.unizg.hr/ipg/
}

\begin{abstract}
In this paper a novel tone mapping operator (TMO) based on the Light Random Sprays Retinex (LRSR) algorithm is presented. TMOs convert high dynamic range (HDR) images to low dynamic range (LDR) images, which is often needed because of the display limitations of many devices. The proposed operator is a local operator, which retains the qualities of the LRSR and overcomes some of its weaknesses. The results of the execution speed and quality tests are presented and discussed and it is shown that on most of the test images the proposed operator is faster and in terms of quality as good as Durand's TMO, one of the currently best TMOs. The $\mathrm{C}++$ source code of the proposed operator is available at http://www.fer.unizg.hr/ipg/resources/color_constancy/.
\end{abstract}

Keywords: HDR, LDR, Light Random Sprays Retinex, Retinex, tone mapping.

\section{Introduction}

The dynamic range of an image is the ratio between the largest and the smallest light intensity in the image. Even though high dynamic range (HDR) images are becoming more available with the recent advances in imaging technologies [18, many standard display devices still require HDR images conversion to low dynamic range (LDR) images by using tone mapping operators (TMOs) in a way that assures visually appealing results. An example of a LDR image obtained by linear mapping of a HDR image and by applying a TMO is shown in Fig. 1. Many TMOs have been proposed [10, 3] 19, 6] 5] 4, [1] and they can be divided into several large main groups: global TMOs, which equally transform same pixel intensity levels, local TMOs, which perform the intensity transformation by taking into account the intensity levels of the pixels in the local surrounding area, and gradient domain based TMOs, which operate on gradients in the luminance domain. Even though the global TMOs are generally faster, the local TMOs tend to give better results [7] [8. TMOs usually operate in the luminance domain instead of the standard RGB domain of the image and for this purpose

A. Elmoataz et al. (Eds.): ICISP 2014, LNCS 8509, pp. 400-408, 2014.

(C) Springer International Publishing Switzerland 2014 


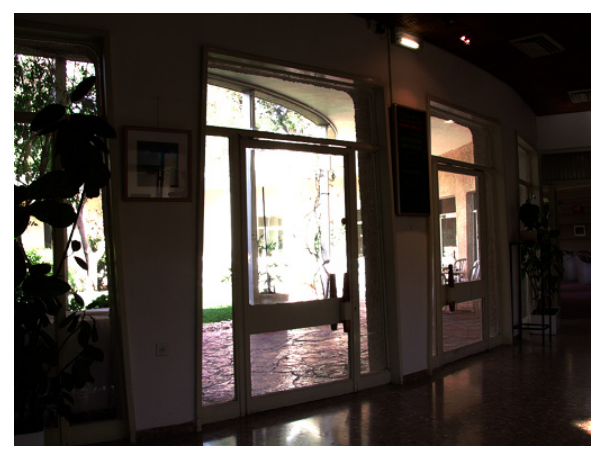

(a)

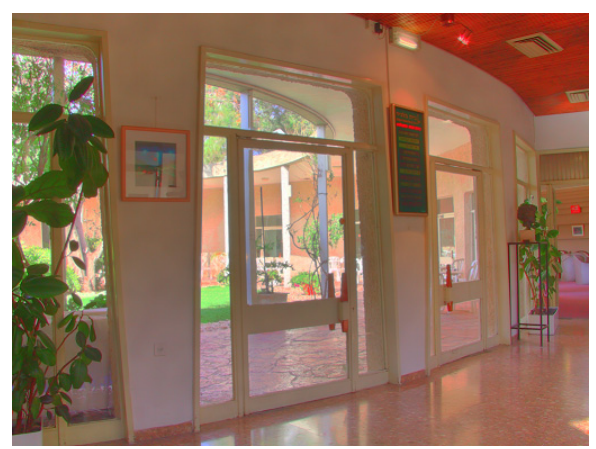

(b)

Fig. 1. Two examples of tone mapping of a HDR image: (a) linear mapping, (b) application of a TMO

the grayscale version of the original image is often used. If the original pixel $p=[r, g, b]^{T}$ has the grayscale value $l$, which after TMO processing becomes $l_{\text {new }}$, then in the resulting image the pixel $p$ is adjusted to become

$$
p_{\text {new }}=\frac{l_{\text {new }}}{l} p=\left[\frac{l_{\text {new }}}{l} r, \frac{l_{\text {new }}}{l} g, \frac{l_{\text {new }}}{l} b\right]^{T} .
$$

The Retinex theory [9] was also used for TMOs. Retinex can be defined as an image enhancement algorithm, which adjusts separate channel values of each pixel by comparing them to the largest values in the local neighborhood. Different Retinex types use different local neighborhoods. In [13] the Multi-Scale Retinex with Color Restoration (MSRCR) [16] was used as a TMO. Recently the improvement of the Random Sprays Retinex (RSR) [15] algorithm called the Light Random Sprays Retinex (LRSR) [2] algorithm was shown to outperform MSRCR and several other algorithms on LDR images in terms of execution speed and resulting image quality. Because LRSR can be shown to be inadequate to process HDR images, we propose a simple, but very effective modification of LRSR, which is shown to give very good results as a TMO.

The paper is structured as follows: In Section 2 a brief description of the LRSR is given, in Section 3 the new tone mapping operator is proposed, in Section 4 it is tested, and the results are presented and discussed.

\section{Light Random Sprays Retinx}

The LRSR algorithm is an improvement of the RSR image enhancement algorithm, which operates on each color channel separately. If $c$ is the chosen channel, $I$ is the original intensity, $R$ is the intensity after applying RSR, $k_{1}$ and $k_{2}$ are averaging kernels, and $O$ is the final result, then LRSR can be summarized as: 


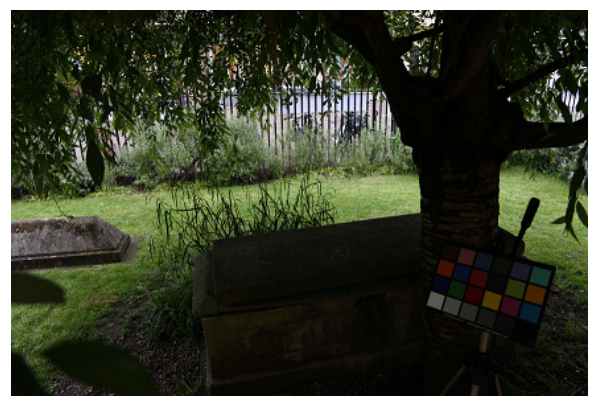

(a)

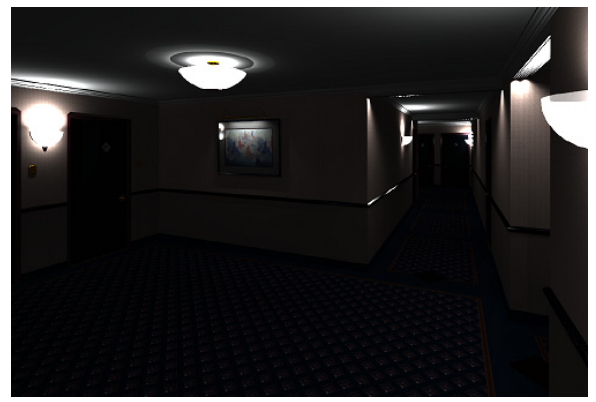

(c)

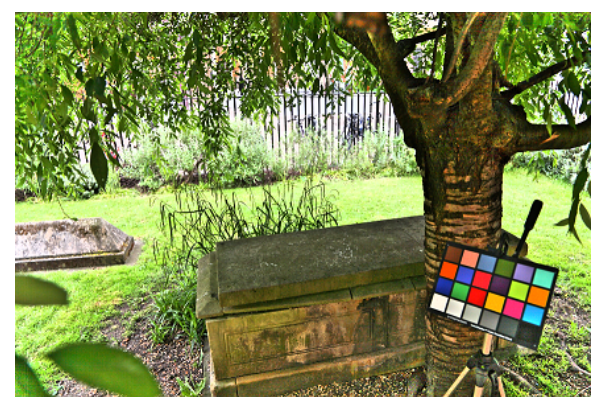

(b)

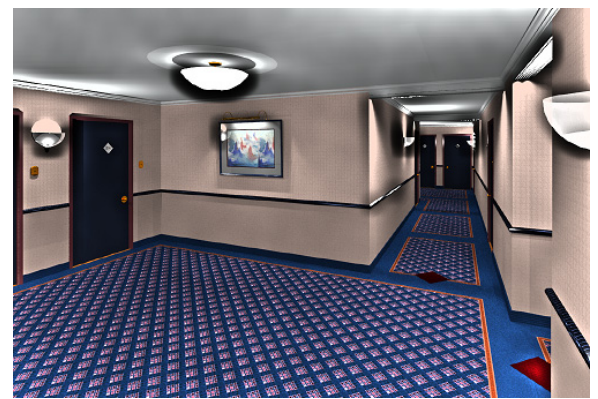

(d)

Fig. 2. LRSR application: (a) the original LDR image, (b) LRSR processed original LDR image, (c) LDR obtained by linear mapping a HDR image, (d) LDR obtained by applying LRSR to a HDR image

$$
\begin{gathered}
C_{c}(i)=\frac{I_{c}(i)}{R_{c}(i)} . \\
C_{c, k_{1}}^{\prime \prime}(i)=\frac{\left(I_{c} * k_{1}\right)(i)}{\left(R_{c} * k_{1}\right)(i)} \\
C_{c, k_{1}, k_{2}}^{*}(i)=\left(C_{c, k_{1}}^{\prime \prime} * k_{2}\right)(i) . \\
O_{c}(i)=\frac{I_{c}(i)}{C_{c, k_{1}, k_{2}}^{*}(i)} .
\end{gathered}
$$

If LRSR is performed on LDR images with unbalanced brightness across the image, it can give good results as shown in Fig. 2b. However, the application of LRSR to HDR images can often lead to halo effects as shown in Fig 2d, 


\section{Proposed Method}

The halo effect appearance is caused by the LRSR noise suppression filtering. When two adjacent areas have very different pixel intensity values, i.e. there is a significant intensity gap between them, one of them will have to be changed more than the other one, i.e. the value of the ratios $\frac{l_{n e w}}{l}$ for pixels in one area will significantly differ from the same ratios for pixels in the other area. In Fig. 2d for example the intensities of the pixels in the area of the lamps should be reduced, i.e. $\frac{l_{\text {new }}}{l}<1$, which is just the opposite than for pixels in the surrounding area. When the filtering of the Eq. 3 and Eq. 4 is performed, the ratios $\frac{l_{n e w}}{l}$ of the adjacent areas influence each other and one of the results is the significant lowering of the rations $\frac{l_{\text {new }}}{l}$ in the area around the lamp, which results in halo effects. In 13, the problem of adjacent areas is solved by detecting edges and in [5] the bilateral filtering is used. Even though these approaches are effective, they introduce a significant execution cost.

We propose to solve the halo effect by globally reducing the intensity gaps and then performing LRSR. The global operation that gave the best results in our experiments is the power function and therefore two new parameters are introduced: $p_{1}$ and $p_{2}$. First, the potential intensity gaps are reduced by raising the grayscale levels to the $\frac{1}{p_{1}}$-th power, then the LRSR is applied and finally the resulting grayscale levels are raised to the $p_{2}$-th power restoring the previous state to a degree. Having $p_{2}<p_{1}$ results in brighter images and reduced contrast, which can be countered by raising the value of the LRSR parameter $n$. Additionally, we introduce two additional parameters $r_{1}$ and $r_{2}$ with meaning that the filterings in Eq. 3 and Eq. 4 are to be iteratively repeated $r_{1}$ and $r_{2}$ times, respectively, thus changing them to

$$
\begin{aligned}
C_{c, k_{1}, r}^{\prime \prime}(i) & =\frac{\left(I_{c} * k_{1}^{* r}\right)(i)}{\left(R_{c} * k_{1}^{* r}\right)(i)} \\
C_{c, k_{1}, k_{2}, r_{1}, r_{2}}^{*}(i) & =\left(C_{c, k_{1}, r_{1}}^{\prime \prime} * k_{2}^{* r_{2}}\right)(i) .
\end{aligned}
$$

where $k_{1}^{* r}$ denotes the convolution power. The effect of such cascade is as if the filtering was performed with a kernel that gives more weight to closer pixels and the complexity is increased only slightly. Some results of the proposed operator are shown in Fig. 3 . Because of the halo effect elimination property, we decided to name the proposed operator Color Badger (CB). The CB pseudocode is given in Algorithm 1 .

TMOs are generally applied to the luminance channel and so is CB. However, instead of correcting all color channels by using the changed luminance channel values, another possibility is to apply CB to all color channels. Such procedure also results in tone mapping, but at the same time local white balancing is performed as shown in Fig. 4, In the experimental tests in the following section $\mathrm{CB}$ is applied only to the luminance channel. The demonstrated application to all channels only had the goal to show the white balancing abilities of CB. 


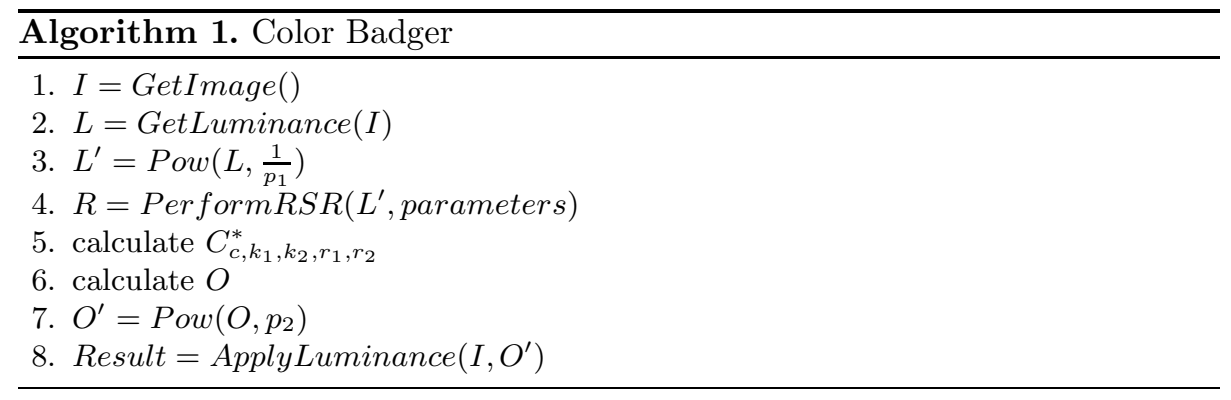

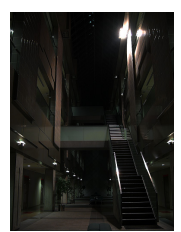
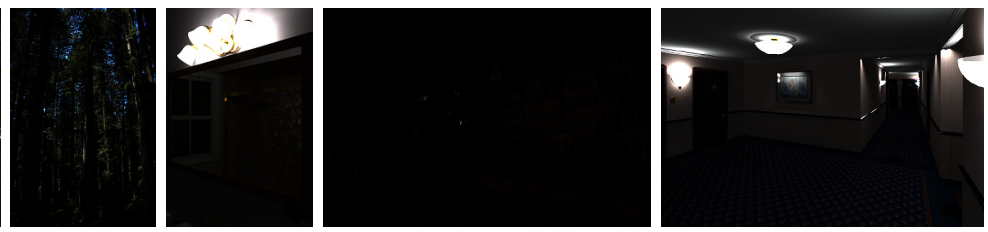

(a)
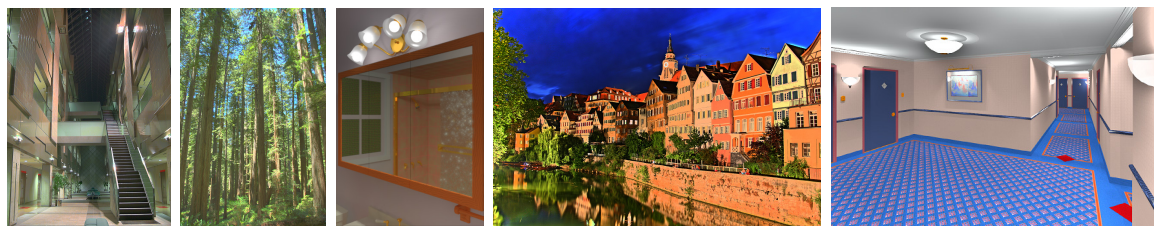

(b)

Fig. 3. Examples of $\mathrm{CB}$ application: (a) linearly mapped HDR images, (b) CB tone mapped HDR images

\section{Experimental Results}

The proposed operator was tested in terms of resulting image quality and execution speed and the results were compared to Durand and Dorsey's bilateral fast filtering technique [5] because it was shown to have one of the best rendering performances in comparison to some other well-known TMOs [7] 8], to LRSR, and to the Drago's 44 and Reinhard's 17] global TMOs, which should have lower result image quality and greater execution speed. The proposed operator was implemented in $\mathrm{C}++$ as well as LRSR and for other mentioned TMOs their highly optimized OpenCV 3.0.0 C++ implementations [1] were used.

\subsection{Quality Test}

For the objective image quality assessment of LDR versions of HDR images the Tone Mapped image Quality Index (TMQI) was used because it is an objective measure reasonably correlated with subjective evaluations of image quality [20]. 


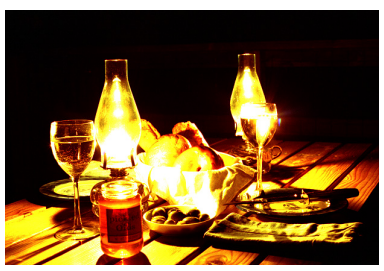

(a)

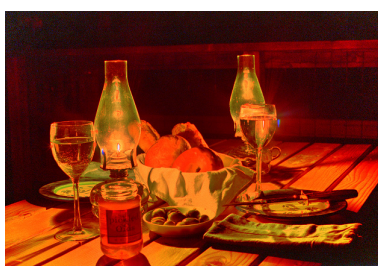

(b)

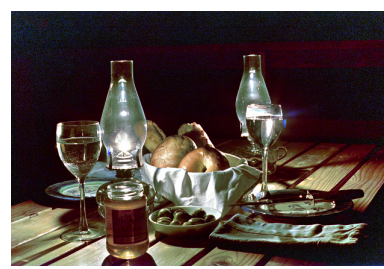

(c)

Fig. 4. Examples of different $\mathrm{CB}$ application: (a) linear mapping, (b) application of CB to the luminance channel, (c) application of CB to all RGB channels

TMQI is a number in the interval $[0,1]$ calculated by comparing the resulting LDR image to the original HDR image and the greater the number, the better the tone mapping result. This measure is based on the multiscale signal fidelity measure and the naturalness measure 20. TMQI can also be used for TMO parameter tuning, which is very useful in automatic parameter setting because the optimal values of TMO parameters are often image-based. Images available at site of the NTUST Compute Graphics Group [14] were used for the quality test by selecting 20 of them with the largest and 20 with the smallest dynamic range. The TMQI of an operator for an image was calculated by taking the maximum TMQI of resulting images obtained by running the operator against the image with many parameter combinations that were chosen to uniformly cover the majority of the practical operator parameter values space. The parameter combinations for CB were formed by choosing the value of $p_{2}$ from $\{1,2, \ldots, 20\}$, the value of $n$ (LRSR inherited individual spray size) from $\{5,6, \ldots, 64\}$, the value of $k_{1}$ and $k_{2}$ from $\{5,11,15,25,51\}$, and the value of $r_{1}$ and $r_{2}$ from $\{1,5,9\}$. The value of $p_{1}$ was fixed to 20 and for the sake of simplicity the values of $k_{1}$ and $k_{2}$ as well as $r_{1}$ and $r_{2}$ were set to be the same. Parameters used for LRSR were the ones in common to CB and LRSR. For Durand and Dorsey's operator the value of the contrast parameter was from $\{0.5,1, \ldots, 25\}$ and the values of the sigma for space and the sigma for color from $\{0.5,1, \ldots, 4\}$. For Drago's operator the value of the bias parameter was from $\{0.01,0.02, \ldots, 1\}$. For Reinhards's operator the value of the intensity parameters was from $\{-8.0,-7.9, \ldots, 8.0\}$ and the values of the light and color adapt parameters were from $\{0.0,0.1, \ldots, 1.0\}$.

Table 1 shows that statistics measures for $\mathrm{CB}$ are better than the ones of other TMOs. This can be attributed to CB's flexibility due to a larger number of parameters, even though the drawback of so many parameters is a more complex tuning. As the best TMQIs for distinct TMOs on selected images were not normally distributed, the Kruskal-Wallis (KW) non-parametric was used [12] to test the statistical significance of the differences between the best TMQIs. Applying it to all tested TMOs shows that there is a statistically significant difference between the results calculated in Table 1 and the KW's box plot is shown in Fig. 5. Even though applying the KW test only to the proposed TMO 
Table 1. TMQI for the proposed and selected TMOs

\begin{tabular}{|c|c|c|c|c|c|}
\hline & CB & Durand & LRSR & Reinhard & Drago \\
\hline mean & $\mathbf{0 . 9 3 1 2}$ & 0.9125 & 0.9079 & 0.8789 & 0.7521 \\
\hline median & $\mathbf{0 . 9 5 3 6}$ & 0.9325 & 0.9109 & 0.8874 & 0.7571 \\
\hline
\end{tabular}

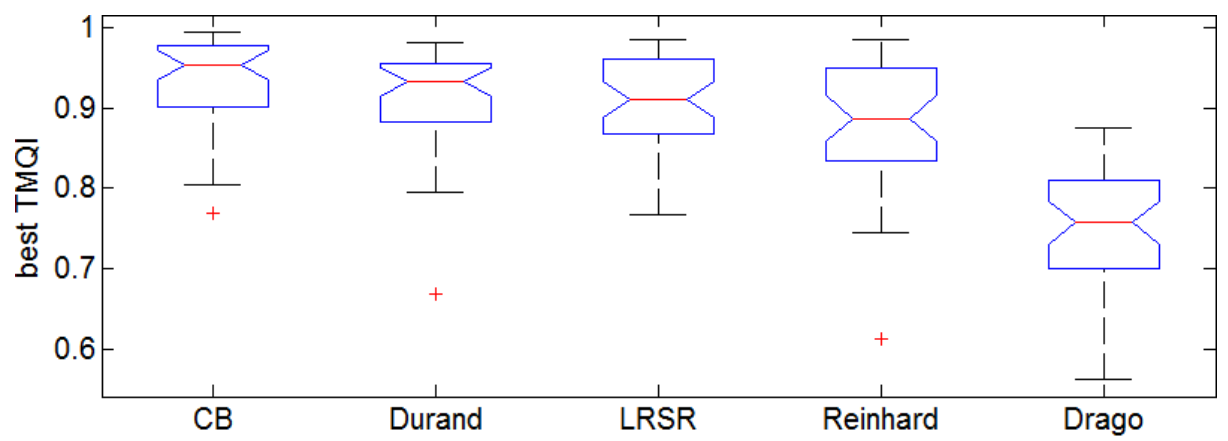

Fig. 5. The box plot for the Kruskal-Wallis test for best TMQIs achieved with different TMOs

and Durand's TMO showed no statistical difference, applying it to compare the proposed TMO with Drago's TMO and the proposed TMO with Reinhard's TMO showed in both cases a $99 \%$ significant difference, which was not the case when Durand's TMO or LRSR were tested in the same way. A comparison of tone mapping results of mentioned algorithms is shown in Fig. 6.
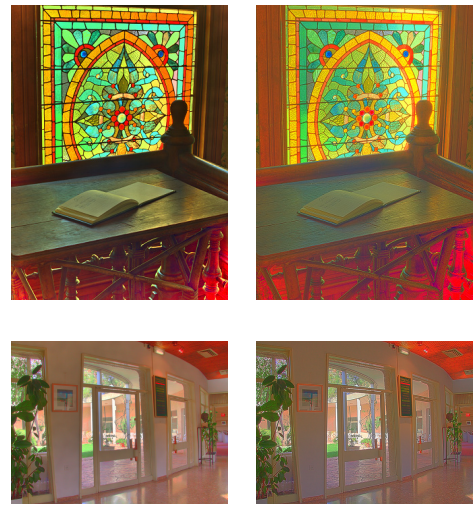

(a)

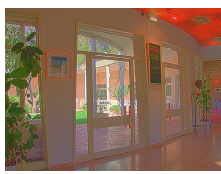

(b)
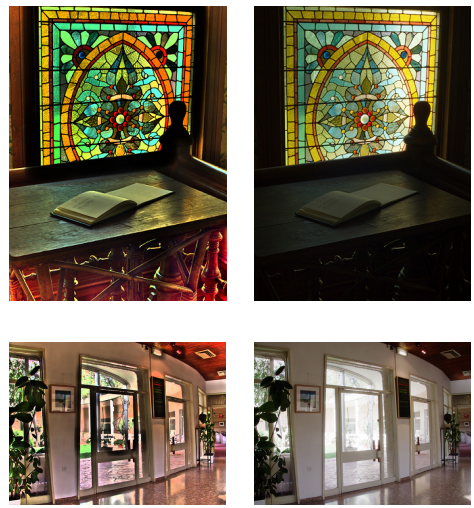

(c)

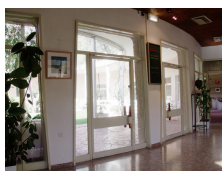

(d)
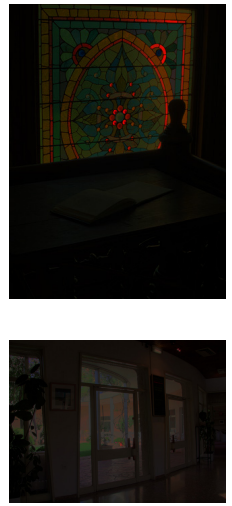

(e)

Fig. 6. Comparison of tone mapping results of several algorithms: (a) CB, (b) Durand's TMO, (c) LRSR, (d) Reinhard's TMO, (e) Drago's TMO 


\subsection{Execution Speed Test}

The execution speed test of the proposed and other operators was performed on a computer with i7-3630QM CPU (only one core was used). All operators were tested on the 40 images used for calculation of Table 1. The LRSR inherited interpolation parameters of the proposed operator were set to maximum value such that the perceptual difference $\Delta E_{a b}^{*}$ for the resulting images obtained without and with interpolation did not exceed 1, i.e. $\Delta E_{a b}^{*}<1$. The combined execution time for the proposed TMO, Durand's TMO, LRSR, Reinhard's TMO, and Drago's TMO on all images was 43.87s, 44.12s, 23.54s, 13.18s, and 12.16s, respectively. It must be mentioned that the code used for Durand's TMO i.e. for bilateral filtering is the highly optimized OpenCV 3.0.0 C++ code using the fast SSE instructions, while the code of the proposed TMO is of prototype quality.

\section{Conclusion}

A new local tone mapping operator was presented and shown to slightly outperform one of the currently best TMOs in terms of execution speed and result quality. Beside the possibility to achieve good tone mapping by applying it to the luminance channel, the proposed operator can also be applied to all RGB channels and used as a local color constancy algorithm for HDR images.

Acknowledgments. The authors would like to thank Dr. Tomislav Petković for his useful remarks. This research has been partially supported by the European Union from the European Regional Development Fund by the project IPA2007/HR/16IPO/001-040514 "VISTA - Computer Vision Innovations for Safe Traffic."

\section{References}

1. HDR imaging (2014), http://docs.opencv.org/master/modules/photo/doc/hdr_imaging.html

2. Banić, N., Lončarić, S.: Light Random Sprays Retinex: Exploiting the Noisy Illumination Estimation. IEEE Signal Processing Letters 20(12), 1240-1243 (2013)

3. Braun, G.J., Fairchild, M.D.: Image lightness rescaling using sigmoidal contrast enhancement functions. Journal of Electronic Imaging 8(4), 380-393 (1999)

4. Drago, F., Myszkowski, K., Annen, T., Chiba, N.: Adaptive logarithmic mapping for displaying high contrast scenes. In: Computer Graphics Forum, vol. 22, pp. 419-426. Wiley Online Library (2003)

5. Durand, F., Dorsey, J.: Fast bilateral filtering for the display of high-dynamic-range images. ACM Transactions on Graphics (TOG) 21(3), 257-266 (2002)

6. Fattal, R., Lischinski, D., Werman, M.: Gradient domain high dynamic range compression. ACM Transactions on Graphics (TOG) 21, 249-256 (2002)

7. Kuang, J., Yamaguchi, H., Johnson, G.M., Fairchild, M.D.: Testing HDR image rendering algorithms. In: Color and Imaging Conference, vol. 2004, pp. 315-320. Society for Imaging Science and Technology (2004) 
8. Kuang, J., Yamaguchi, H., Liu, C., Johnson, G.M., Fairchild, M.D.: Evaluating HDR rendering algorithms. ACM Transactions on Applied Perception (TAP) 4(2), 9 (2007)

9. Land, E.H.: The retinex. American Scientist 52(2), 247-264 (1964)

10. Larson, G.W., Rushmeier, H., Piatko, C.: A visibility matching tone reproduction operator for high dynamic range scenes. IEEE Transactions on Visualization and Computer Graphics 3(4), 291-306 (1997)

11. Mantiuk, R., Daly, S., Kerofsky, L.: Display adaptive tone mapping. ACM Transactions on Graphics (TOG) 27, 68 (2008)

12. Maxwell, S., Delaney, H.: Designing Experiments and Analyzing Data: A Model Comparison Perspective. No. s. 1 in Designing Experiments and Analyzing Data: A Model Comparison Perspective, Lawrence Erlbaum Associates (2004), http://books.google.hr/books?id=gKZbD3lL88AC

13. Meylan, L., Susstrunk, S.: High dynamic range image rendering with a retinexbased adaptive filter. IEEE Transactions on Image Processing 15(9), 2820-2830 (2006)

14. NTUST Compute Graphics Group: HDR (2014), http://graphics.csie.ntust.edu.tw/pub/HDR/

15. Provenzi, E., Fierro, M., Rizzi, A., De Carli, L., Gadia, D., Marini, D.: Random spray retinex: A new retinex implementation to investigate the local properties of the model. IEEE Transactions on Image Processing 16(1), 162-171 (2007)

16. Rahman, Z.U., Jobson, D.J., Woodell, G.A.: Retinex processing for automatic image enhancement. Journal of Electronic Imaging 13(1), 100-110 (2004)

17. Reinhard, E., Devlin, K.: Dynamic range reduction inspired by photoreceptor physiology. IEEE Transactions on Visualization and Computer Graphics 11(1), 13-24 (2005)

18. Reinhard, E., Heidrich, W., Debevec, P., Pattanaik, S., Ward, G., Myszkowski, K.: High dynamic range imaging: Acquisition, display, and image-based lighting. Morgan Kaufmann (2010)

19. Reinhard, E., Stark, M., Shirley, P., Ferwerda, J.: Photographic tone reproduction for digital images. ACM Transactions on Graphics (TOG) 21, 267-276 (2002)

20. Yeganeh, H., Zhou, W.: Objective Quality Assessment of Tone Mapped Images. IEEE Transactions on Image Processing 22(2), 657-667 (2013) 